\title{
Stem Rot on Adzuki Bean (Vigna angularis) Caused by Rhizoctonia solani AG 4 HGI in China
}

\author{
Suli Sun ${ }^{1 \dagger}$, Changjian Xia ${ }^{1 \dagger}$, Jiqing Zhang ${ }^{1}$, Canxing Duan ${ }^{1}$, Xiaoming Wang ${ }^{1}$, Xiaofei Wu ${ }^{1}$, Suk-Ha Lee ${ }^{2}$ and \\ Zhendong $\mathrm{Zhu}^{1 *}$ \\ ${ }^{I}$ The National Key Facility for Crop Gene Resources and Genetic Improvement, Institute of Crop Science, Chinese Acad- \\ emy of Agricultural Sciences, 12 Zhongguancun South Street, Beijing 100081, People's Republic of China \\ ${ }^{2}$ Department of Plant Science and Research Institute for Agriculture and Life Sciences, Seoul National University, Seoul \\ 151-921, Korea
}

(Received on July 16, 2014; Revised on October 8, 2014; Accepted on November 2, 2014)

\begin{abstract}
During late August and early September 2011, stem rot symptoms were observed on adzuki bean plants (Vigna angularis) growing in fields located in Beijing and Hebei Province, China, respectively. In this study, four isolates were obtained from infected stems of adzuki bean plants. Based on their morphology, and sequence and polymerase chain reaction-restriction fragment length polymorphism (PCR-RFLP) analyses of the ribosomal DNA internal transcribed spacers (rDNA-ITS) region, the four isolates were identified as Rhizoctonia solani in anastomosis group (AG) 4 HGI. Pathogenicity tests showed that all isolates were strongly pathogenic to adzuki bean and resulted in serious wilt symptoms which was similar to observations in the fields. Additionally, the isolates infected several other crops and induced related rot on the roots and basal stems. To our knowledge, this is the first report of Rhizoctonia solani AG 4 HGI causing stem rot on adzuki bean.
\end{abstract}

Keywords : adzuki bean, anastomosis group, PCR-RFLP, Rhizoctonia solani, stem rot

Adzuki bean (Vigna angularis), an important edible legume crop, has attracted more and more attention from scientists and consumers because of its high nutritional and medicinal value. As of 2011, China had the largest planting area at 250,000 ha and the greatest yield of approximately $300,000 \mathrm{t}$ of adzuki bean (Wang et al., 2013). However, one of the main constraints on adzuki bean production in

\footnotetext{
"These authors contributed equally to this work.

*Corresponding author.

Phone) +86-10-8210-9609, FAX) +86-10-8210-9608

E-mail) zhuzhendong@caas.cn
}

China in recent years has been the occurrence of diseases in the fields (Wang et al., 2000).

During late August and early September 2011, many wilting plants were observed in fields of adzuki bean in Beijing and Hebei Province, China, respectively. The wilting plants had girdled stem rot on the lower stems. Initially, the infected stems showed water-soaked lesions at the infection point (Fig. 1a); however, the lesions rapidly expanded up and down. The stem became girdled, dry and discolored as the disease developed (Fig. 1b). Finally, the infection caused withering and death of plants (Fig. 1c, 1d). Similar symptoms had been recorded on adzuki bean in China previously. At that time Rhizoctonia solani was thought to be the causal agent of the disease, but it was not confirmed (Wang et al., 2000). Therefore, this study was designed to identify and characterize the causal pathogen of stem rot on adzuki bean plants based on colony morphology combined with molecular techniques, including sequence and PCR-RFLP analyses of the rDNA-ITS region. Additionally, we determined the pathogenicity of the causal agent to several crops, including adzuki bean.

Adzuki bean plants showing typical symptoms of stem rot were collected from fields located in Beijing and Hebei Province, China, during the 2011 growing season. The causal pathogen was obtained from infected stems using the tissue isolation method. Briefly, advancing lesions of infected stems were cut into small sections $(3 \mathrm{~mm} \times 3 \mathrm{~mm})$. The small pieces of stem tissue were surface-disinfected in $70 \%$ ethanol for $30 \mathrm{~s}, 1 \%$ sodium hypochlorite for $5 \mathrm{~min}$, rinsed three times in sterile distilled water, and then placed on $2 \%$ water agar acidified with lactic acid ( $\mathrm{pH} 4.5)$. The resulting colonies were examined for typical Rhizoctonia growth after incubation for 1 to 2 days at $25^{\circ} \mathrm{C}$ with a 12 -h light/dark regime. Hyphal tips excised from branches were transferred to potato-dextrose agar (PDA; AoBoXing, Bio- 
tech, Beijing, China) acidified with lactic acid and supplemented with $50 \mathrm{mg} / \mathrm{l}$ streptomycin sulfate to inhibit bacterial growth (Sigma-Aldrich, St. Louis, MO, USA). All the plates were incubated at $25^{\circ} \mathrm{C}$ for 3 to 4 days. The obtained isolates were stored on PDA slants at $4^{\circ} \mathrm{C}$ for further use.

Four isolates, XDR1 and XDR2 from Hebei Province, and XDR3 and XDR4 from Beijing, were selected to determine their identities. Young hyphae of the isolates were stained with $0.03 \%$ safranin-O and $3 \% \mathrm{KOH}$ aqueous solution according to the protocol of Bandoni (1979). The numbers of nuclei in the stained hyphae were examined using an Olympus CX31 microscope at $400 \times$ to determine binucleate or multinucleate status. The four isolates were further characterized by sequence and PCR-RFLP analyses of the rDNA-ITS region following the procedures described by Guillemaut et al. (2003) and Pannecoucque et al. (2008). Briefly, for the sequence analysis of the rDNA-ITS region, DNA of the four isolates was extracted and amplified with universal primers ITS1/ITS4 (White et al., 1990). The PCR products were further analyzed by sequencing the ITS region. The obtained sequences were blasted against the GenBank (National Center for Biotechnology Information, NCBI) database using Blastn. For the PCR-RFLP analysis of the rDNA-ITS region, genomic DNA from each of the four isolates was amplified with primers RS1/RS4, and the resulting PCR products were further analyzed by RFLP using four restriction enzymes, MunI, MseI, AvaII and HincII (Guillemaut et al., 2003).

The pathogenicity of four $R$. solani isolates was tested. Because $R$. solani has a wide range of hosts, the pathogenicity test was performed not only on the original host, adzuki bean (cv. Baihong 1 and line BH06-169-6), but also on other crops, including mung bean (Vigna radiata), common bean (Phaseolus vulgaris), cowpea (V. unguiculata), cotton (Anemone vitifolia), towel gourd (Luffa cylindrica), maize (Zea mays) and wheat (Triticum aestivum). All of the tested crops were sown into 250-ml paper cups (five disinfected seeds/cup) filled with sand that had been sterilized by autoclaving at $121^{\circ} \mathrm{C}$ for $1 \mathrm{~h}$. The planted cups were randomly distributed on a greenhouse bench under a temperature of $25 \pm 2^{\circ} \mathrm{C}$. Inoculums of the four isolates were cultured in potato-dextrose broth (PDB) $(100 \mathrm{ml}$ in 250-ml flasks) with constant shaking at $150 \mathrm{rpm}$ for 7 days at $26^{\circ} \mathrm{C}$. The resulting cultures were homogenized for $30 \mathrm{~s}$ in a Waring blender, and the concentration of the mycelial suspension was adjusted to approximately $1.0 \times 10^{6} \mathrm{cfu} / \mathrm{ml}$. Then, 10-day-old seedlings were inoculated by pouring the mycelial suspension around the base of the plants $(5 \mathrm{ml} /$ plant). A negative control was treated in the same manner using sterile PDB. Eight days after inoculation, the disease severity of the seedlings was scored using a scale range described by Li (1991). The above experiments were performed twice with three replicates.

Four isolates (XDR1 and XDR2 from Hebei Province, and XDR3 and XDR4 from Beijing) were obtained from stems of adzuki bean plants exhibiting stem rot symptoms. The four isolates grew rapidly on PDA plates, producing $2.5 \mathrm{~cm}$ and $7.0 \mathrm{~cm}$ diameter colonies at 1 and 2 days after inoculation, respectively. After 3 days of incubation, the colonies overgrew the $9.0-\mathrm{cm}$ diameter Petri-dishes. The colonies of isolates growing on PDA were whitish initially and turned brown to dark brown after 4 days (Supplementary Fig. 1a). Brown sclerotia were observed on the lids of Petri-dishes at 9 days after inoculation. Hyphae of isolates presented rectangular or acute branches with constricted bases (Supplementary Fig. 1b). Nuclear staining of hyphae using safranin-O revealed that the isolates were multinucleate (Supplementary Fig. 1c). All morphological characteristics and staining reactions indicated that the four isolates belonged to the multinucleate species $R$. solani.

For further confirmation, sequence analyses of the four isolates' rDNA-ITS regions were performed. The obtained
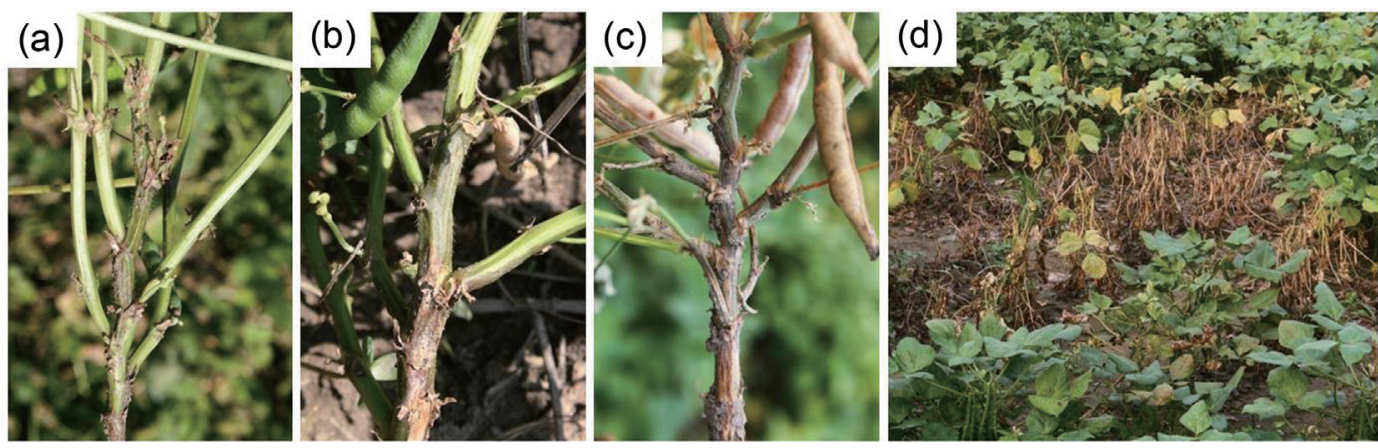

Fig. 1. Stem rot symptoms caused by Rhizoctonia solani AG 4 HGI observed on adzuki bean plants in the field. (a) Early symptoms on an infected stem; (b) Developing symptoms on an infected stem; (c) Late symptoms on an infected stem with sclerotial formation; (d) Lots of seriously withered plants. 
sequences from the four isolates, XDR1, XDR2, XDR3 and XDR4, were submitted to the NCBI database and accession numbers (Nos) KC405625, KC405626, KC405627 and KC405628, respectively, were assigned. A Blastn analysis in GenBank of each obtained sequence showed that the four isolates were highly homologous with many $R$. solani strains and the identified subgroups of $R$. solani AG 4 HGI isolates. Our four isolates, XDR1, XDR2, XDR3 and XDR4, showed the higher similarities (95 to 99\%) with reported $R$. solani AG 4 HGI isolates from Cynanchum paniculatum (NCBI GenBank accession No. JQ343830) and Vincetoxicum pycnostelma (JQ669933) in China; from unidentified host (AB000007) in Japan; from Phaseolus vulgaris (HE805679) in Turkey; and from stem rot of tomato (AY154307) in Brazil (Kuramae et al., 2003). Moreover, the homology between the two isolates (XDR1 and XDR2, or XDR3 and XDR4) from the same location was higher than between isolates from different locations. To confirm the subgroups of the four $R$. solani isolates, the approximately $540 \mathrm{bp}$ PCR products from the rDNA-ITS regions were digested with the restriction endonuclease enzymes, MunI, HincII, AvaII and MseI. The RFLP patterns were observed on an agarose gel (Fig. 2). The amplification products of the rDNA-ITS regions obtained using RS1/4 had only one accessible MunI, HincII and AvaII restriction sites, but $M s e$ I had multiple accessible recognition sites. The RFLP analysis of the PCR products from the Beijing isolates XDR3 and XDR4 showed two bands of approximately $340 \mathrm{bp}$ and $200 \mathrm{bp}$ when digested by MunI. However, the PCR amplification products of the Hebei Province isolates XDR1 and XDR2 showed additional bands when digested by MunI (Fig. 2). This result was consistent with the previous observations of sequence heterogeneity (Pan- necoucque et al., 2008; Pannecoucque and Höfte, 2009; Strausbaugh et al., 2011). PCR products were digested by HincII and AvaII into two bands of approximately $330 \mathrm{bp}$ and $210 \mathrm{bp}$, and $490 \mathrm{bp}$ and $50 \mathrm{bp}$, respectively (Guillemaut et al., 2003; Pannecoucque et al., 2008). Digestion by MseI at several recognition sites resulted in multiple smaller fragments. They appear to be approximately $220 \mathrm{bp}, 140 \mathrm{bp}, 80$ bp and $60 \mathrm{bp}$ bands in agarose gels (Fig. 2). It is possible that smaller fragments were not resolved in the agarose gel based on the total length of the PCR products. Thus, bands of less than $40 \mathrm{bp}$ were not taken into consideration, which is in agreement with a previous study (Guillemaut et al., 2003). Based on the size of the restriction fragments, RFLP types generated by the digestion of four enzymes, MseI, AvaII, MunI and HincII, are I, E, A, and A, respectively, and these RFLP types are specific to $R$. solani AG 4 HGI according to the classes of restriction patterns described by Guillemaut et al. (2003). Therefore, the RFLP analysis also classified the four isolates into AG 4 HGI subgroups. The sequence and PCR-RFLP analyses of the rDNA-ITS region provided further support for the isolates belonging to $R$. solani AG 4 HGI.

The pathogenicity tests of the four isolates were performed on 10-day-old seedlings of adzuki bean and 7 other crops by inoculating the basal stems. The four isolates induced rot symptoms on roots and basal stems not only on the original host, adzuki bean, but also on most of the other crops tested (Supplementary Figs. 2 and 3). No significant differences in pathogenicity were observed on the adzuki bean among the four $R$. solani AG 4 HGI isolates. All of the diseased plants showed similar symptoms on the basal stems with observations in the fields 8 days after inoculation. Lastly, the four isolates resulted in serious wilt

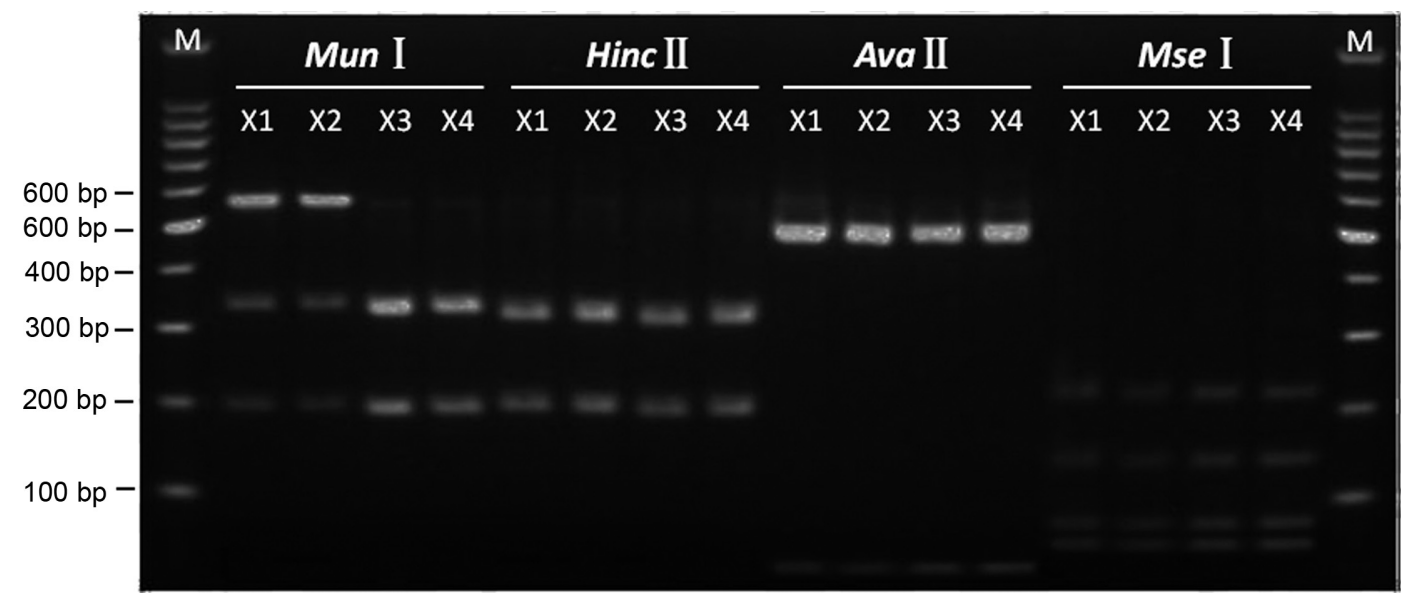

Fig. 2. PCR-RFLP patterns of the rDNA-ITS region of four fungal isolates, XDR1, XDR2, XDR3 and XDR4, digested with MunI, HincII, AvaII and MseI restriction endonucleases and resolved in a 1.5\% agarose gel. XDR1 and XDR2 were isolated in Hebei Province, and XDR3 and XDR4 were isolated in Beijing. (M: DNA markers; X1: XDR1; X2: XDR2; X3: XDR3; X4: XDR4). 
symptoms due to basal stem rot and root rot on adzuki bean and additional tested crops, including mung bean, common bean, cowpea, towel gourd and cotton (Supplementary Figs. 2 and 3). However, the tested cereal crops, wheat and corn, showed mild symptoms (Supplementary Figs. 2 and 3). The four isolates were also re-isolated from respective symptomatic lesion regions in all tested crops, in accordance with Koch's postulates. Neither symptoms appeared nor pathogens were obtained from the control plants. Our results indicate that the isolates have the potential to cause damage not only to beans but also to other crops.

The correct identification of the causal pathogen is critical to the appropriate selection of isolates for resistancescreening programs and disease control, as well as for intercropping or crop rotation strategies (Fenille et al., 2002). In the present study, the causal pathogen of stem rot of adzuki bean was confirmed as $R$. solani AG 4 HGI by combining colony morphology and molecular identification techniques. The latter includes sequence and PCRRFLP analyses of the rDNA-ITS region, which have been considered the criteria to identify $R$. solani at the AG and subgroup levels (Boysen et al., 1996; Guillemaut et al., 2003; Pannecoucque et al., 2008).

In the PCR-RFLP analysis, it is interesting that variation in PCR-RFLP patterns was observed using the restriction enzymes MunI among the four isolates of $R$. solani AG 4 HGI. Two different digestion patterns appeared in the agarose gel (Fig. 2). This result revealed that sequence heterogeneity exists within the isolates. This phenomenon of heterogeneous digestion patterns in rDNA-ITS region of $R$. solani isolates has been observed in recent studies by other researchers (Pannecoucque et al., 2008; Pannecoucque and Höfte, 2009; Strausbaugh et al., 2011). In addition, the results of direct sequencing revealed that homology within isolates from the same geographical region, XDR1 and XDR2 or XDR3 and XDR4, is higher than between two isolates from different regions. This corroborates results on the genetic structure of populations of $R$. solani AG 4 (Haratian et al., 2013).

In previous studies, $R$. solani AG 4 HGI was reported as a main pathogen causing major damping-off, and root and hypocotyl rot in beans (Nerey et al., 2010). In China, the pathogen $R$. solani AG 4 HGI was observed to cause the head rot of cabbage (Brassica oleracea var. capitata) and the web blight of the snap bean (Phaseolus vulgaris) (Yang et al., 2007). Additionally, $R$. solani AG 4 HGI and the other two subgroups, HGII and HGIII, can cause stem rot or stem canker on tomato, castor bean, green amaranth and Chinese amaranth (Basseto et al., 2008; Kuramae et al., 2003; Yang et al., 2005). Our results and previous stud- ies show that $R$. solani AG 4 HGI can cause various types of diseases in a wide range of hosts. Thus, to reduce Rhizoctonia stem rot on adzuki bean, crop rotational strategies should be given further consideration.

Recently, hypocotyl rot of adzuki bean caused by species R. solani AG-1 IB was observed in Japanese fields (Misawa and Komatsu, 2011). To the best of our knowledge, there are no reports to date describing $R$. solani AG 4 HGI occurring on adzuki bean. Thus, this is the first report of $R$. solani AG 4 HGI infecting adzuki bean and causing stem rot. Our results indicated that stem rot caused by $R$. solani AG 4 HGI is a new threat to adzuki bean production and adzuki bean might be acting as a reservoir and spreading this pathogen to other economically important crops in China.

\section{Acknowledgment}

This research was supported by a grant from the China Agriculture Research System (CARS-09).

\section{References}

Bandoni, R. J. 1979. Safranin O as a rapid nuclear stain for fungi. Mycologia 71:873-874.

Basseto, M. A. 2008. First report of Rhizoctonia solani AG 4 HGII attacking castor bean plants (Ricinus communis) in Brazil and evaluation of two castor bean cultivars for resistance to damping-off. Austral. Plant Dis. Notes 3:121-123.

Boysen, M., Borja, M., Del Salazar, M. C. and Rubio, V. O. 1996. Identification at strain level of Rhizoctonia solani AG 4 isolates by direct sequence of asymmetric of the ITS regions. Curr. Genet. 29:174-181.

Fenille, R. C., de Souza, N. L. and Kuramae, E. E. 2002. Characterization of Rhizoctonia solani associated with soybean in Brazil. Eur. J. Plant Pathol. 108:783-792.

Guillemaut, C., Edel-Herman, V., Comporota, P., Alabouvette, C., Richard-Molard, M. and Steinberg, C. 2003. Typing of anastomosis groups of Rhizoctonia solani by restriction analysis of ribosomal DNA. Can. J. Microbiol. 49:556-568.

Haratiana, M., Safaiea, N., Sharifnabib, B., Mahmudic, S. B. and Arianab, A. 2013. Genetic structure of populations of Rhizoctonia solani AG-4 from five provinces in Iran. Plant Pathol. 62:649-656.

Kuramae, E. E., Buzeto, A. L., Ciampi, M. B. and Souza, N. L. 2003. Identification of Rhizoctonia solani AG 1-IB in lettuce, AG 4 HG-I in tomato and melon, and AG 4 HG-III in broccoli and spinach, in Brazil. Eur. J. Plant Pathol. 109:391-395.

Li, Y. L. 1991. Rhizoctonia root rot of mungbean. In: Methods used in the evaluation of pest resistant potentialities in food crop germplasm resources eds. by Q. A. Wu, K. G. Liang and J. Cao, pp. 58-59. Beijing: China Agriculture Press, Led. (in 
Chinese).

Misawa, T. and Komatsu, T. 2011. Occurrence of Rhizoctonia root rot of soybean caused by Rhizoctonia solani AG-2-2IV and adzuki bean caused by $R$. solani AG-1 IB in commercial fields of Hokkaido. Annu. Rep. Soc. Plant Prot. North Jpn. 62:50-54.

Nerey, Y., Beneden, S. V., França, S. C., Jimenez, A., Cupull, R., Herrera, L. and Höfte, M. 2010. Influence of soil type and indigenous pathogenic fungi on bean hypocotyls rot caused by Rhizoctonia solani AG 4 HGI in Cuba. Soil Biol. Biochem. 42:797-803.

Pannecoucque, J. and Höfte, M. 2009. Detection of rDNA ITS polymorphism in Rhizoctonia solani AG 2-1 isolates. Mycologia 101:26-33.

Pannecoucque, J., Van Beneden, S. and Höfte, M. 2008. Characterization and pathogenicity of Rhizoctonia isolates associated with cauliflower in Belgium. Plant Pathol. 57:737-746.

Strausbaugh, C. A., Eujayl, I. A., Panella, L. W. and Hanson, L. E. 2011. Virulence, distribution and diversity of Rhizoctonia solani from sugar beet in Idaho and Oregon. Can. J. Plant Pathol. 33:210-226.

Wang, L. X., Cheng, X. Z. and Wang, S. H. 2013. Review on genetic study and application of adzuki bean (Vigna angularis) germplasm. J. Plant Genet. Resour. 14:440-447.

Wang, X. M., Jin, D. S., Redden, R., Wang, S. M., Lawrence, P., Zong, X. X., Hu, J. P. and Cao, Y. S. 2000. Adzuki bean diseases and insect pests. Beijing: China Agricultural Science and Technique Press, Led. (in Chinese).

White, T. J., Bruns, T., Lee, S. and Taylor, J. W. 1990. Amplification and direct sequencing of fungal ribosomal RNA genes for phylogenetics. In: PCR Protocols: A Guide to Methods and Applications eds. by M. A. Innis, D. H. Gelfand, J. J. Sninsky and T. J. White, pp. 315-322. New York: Academic Press, Inc.

Yang, G. H., Chen, J. Y. and Pu, W. Q. 2007. First report of head rot of cabbage and web blight of snap bean caused by Rhizoctonia solani AG-4 HGI. Plant Pathol. 56:351.

Yang, G. H., Chen, H. R., Naito, S. and Ogoshi, A. 2005. Characterization of Rhizoctonia solani AG 4 HG-III causing stem canker and wirestem on green amaranth and Chinese amaranth. J. Phytopathol. 153:185-187.

Zhu, Z. D. and Wang, X. M. 2003. Identification of pathogen causing Phytophthora stem rot of adzuki bean and screening for resistant germplasm. ACTA Phytophylacica Sinica 30:289-294. 